\title{
POLUIÇÃO HARMÓNICA EM INSTALAÇÕES ELÉTRICAS INDUSTRIAIS
}

\section{Introdução}

Qualidade de Energia Elétrica (QEE) pode ser definida como a ausência relativa de perturbações que afetem a correta operação de um qualquer equipamento ou instalação elétrica.

As empresas distribuidoras de energia elétrica encaram hoje a QEE como um fator fundamental, sendo mesmo motivo de preocupação, dadas as necessidades dos consumidores atuais e as eventuais situações que podem conduzir a problemas de complexa resolução.

Com o evoluir da tecnologia, são atualmente muito utilizados equipamentos de controlo e potência baseados em eletrónica de estado sólido. Estes tipos de equipamentos, para além de "poluir" a rede elétrica, também são bastante sensíveis a fatores relacionados com a qualidade da energia elétrica.

Se inicialmente a qualidade da energia elétrica era considerada num domínio relativamente restrito, hoje esse domínio está severamente alargado. Assim, preocupações específicas apenas relativas à continuidade de serviço são hoje insuficientes para as necessidades dos consumidores atuais. Neste cenário, para além ter-se de garantir os devidos níveis de tensão e frequência no fornecimento de energia elétrica, também tem de ser devidamente avaliada a situação correspondente ao nível de poluição harmónica.

Embora na distribuição e transmissão de energia não sejam conhecidos problemas relevantes relacionados com a qualidade da energia, na indústria a situação é diferente. Associado a uma completa incerteza da causa, e por vezes do causador, da não existência de energia com qualidade, os prejuízos económicos resultantes são muito elevados, quer em termos de paragem de unidades de produção, quer em termos de equipamento danificado.
Apesar de este problema atingir de forma severa a generalidade do setor industrial, realçam-se alguns subsetores, hoje identificados como críticos:

- Indústria de semicondutores;

- Indústria de papel;

- Indústria automóvel (soldadura);

- Indústrias com consumo elevado de energia elétrica.

Em Portugal a maioria das empresas não têm as suas instalações elétricas preparadas para lidar com estes problemas e as respetivas consequências relativas à ausência de qualidade da energia elétrica. A ausência de indicadores da qualidade de energia e a incompleta monitorização da rede, torna muito difícil identificar a causa de uma diversidade de problemas resultantes da falta de qualidade da energia elétrica.

O facto das instalações elétricas não estarem em condições de fazer face aos problemas da qualidade de energia, isto não se deve necessariamente a erros na conceção inicial da instalação, mas devido a alterações nos tipos de equipamentos entretanto utilizados pelas empresas:

- Equipamentos mais sensíveis a perturbações, por incluírem sistemas de controlo baseados em componentes eletrónicos que não são concebidos para funcionarem em ambientes "poluídos";

- Equipamentos poluidores da rede elétrica, em termos de geração e propagação de componentes harmónicas.

Os equipamentos deste último ponto relacionam-se com a utilização da eletrónica de potência em larga escala na indústria, como os conversores eletrónicos, sistemas de retificação, controladores lógicos programáveis (PLCs), 
variadores de velocidade para motores de corrente contínua

e corrente alternada, lâmpadas de descarga, etc. Em resultado disso, é possível observar-se uma crescente deterioração das formas de onda de corrente e tensão dos sistemas de energia elétrica. A deformação da forma de onda destas grandezas, que idealmente variam no tempo de forma sinusoidal, leva-nos à problemática das componentes harmónicas nas instalações elétricas em particular [1].

\section{Qualidade de EE}

Como atesta a norma EN 50160, a qualidade de energia elétrica é definida como a ausência de qualquer perturbação que afete a operação de um qualquer equipamento recetor. Sendo assim, considerando ainda este normativo, um problema de Qualidade da Energia caracteriza-se como qualquer distúrbio ou ocorrência manifestada nos níveis e em formas de onda de tensão ou corrente, que possam resultar em insuficiência, má operação, falha ou defeito permanente em equipamentos de um sistema elétrico.
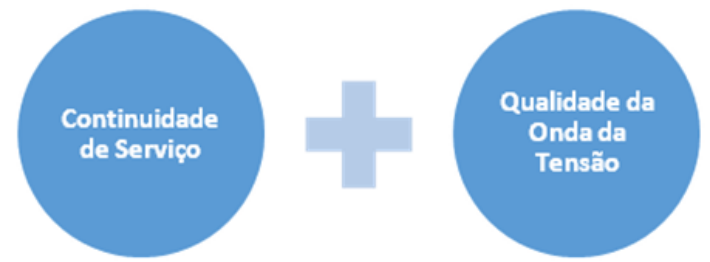

Figura 1. Perceção e Complexidade na Definição de QEE

A definição apresentada na norma refere-se à qualidade da onda de tensão como sinónimo da qualidade da energia elétrica. Assim, esta pode ser descrita como a disponibilidade de energia elétrica com forma de onda sinusoidal pura, sem alterações na amplitude, emanando de uma fonte de potência infinita. De facto, do ponto de vista académico, qualquer desvio na característica destes parâmetros é considerado um problema envolvendo a QEE. Esta é apenas uma definição, que não sendo universal dá uma ideia rápida do que pretende refletir, limitando-se no entanto à qualidade da onda de tensão. Uma definição mais ampla, que tem em conta pontos adicionais da energia elétrica como produto e serviço, atende ao seguinte:

- Continuidade de serviço (Fiabilidade);
- $\quad$ Ausências de interrupções;

- Qualidade da onda;

- Amplitude constante com valor nominal;

- Frequência constante;

- Sistema de tensões equilibrado e simétrico;

- Formas de onda sinusoidais;

- Qualidade comercial;

- Atendimento (presencial ou telefónico);

- Informação disponibilizada (Contratos, opções, serviços, reclamações, faturação, etc.);

- Padrões para a qualidade comercial.
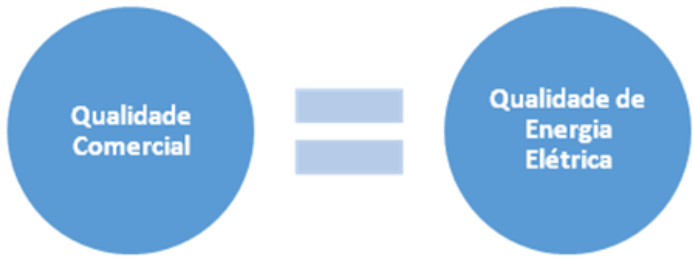

A degradação da QEE pode ocorrer devido a vários tipos de distúrbios elétricos. Estes distúrbios podem ter origem no fornecimento da energia através da rede elétrica ou no consumidor, dependendo dos equipamentos que este tenha instalado.

Os indicadores de qualidade são referidos em vários documentos para avaliar a qualidade da onda de tensão (NP EN 50160, normas CEI, IEEE, etc.). Os referidos pela NPEN50160 descrevem as características técnicas fundamentais da energia elétrica, como:

- Frequência;

- Amplitude da tensão de alimentação;

- Variações da tensão de alimentação; 
- Variações rápidas da tensão de alimentação;

- Amplitude das variações rápidas;

- Severidade da tremulação (flicker);

- Cavas da tensão de alimentação;

- Interrupções breves da tensão de alimentação;

- Interrupções longas da tensão de alimentação;

- Sobretensões temporárias entre os condutores ativos e a terra;

- Sobretensões transitórias entre os condutores ativos e a terra;

- Desequilíbrio das tensões de alimentação;

- Tensões harmónicas;

- Tensões inter-harmónicas;

- Transmissão de sinais de informação na rede.

\section{Harmónicas}

O termo harmónico vem da física, mais especificamente do estudo dos movimentos ondulatórios. Quando uma partícula, ou uma onda, se propaga oscilando periodicamente em torno de uma posição de equilíbrio, esse movimento pode ser traduzido matematicamente por funções sinusoidais, denominando-se "movimento harmónico".
Tecnicamente uma harmónica é uma componente de onda periódica cuja frequência é um múltiplo inteiro da frequência fundamental. Isso pode ser facilmente visualizado na figura 2. Nela, apresentam-se três formas de onda distintas. Uma onda sinusoidal considerada à frequência de $(60 \mathrm{~Hz})$ e duas outras representando determinadas ondas harmónicas. Como para a segunda e a terceira onda os ciclos repetem-se, respetivamente, 3 e 5 vezes no mesmo período de tempo em que a onda fundamental descreve apenas um ciclo, verifica-se que elas representam as harmónicas de 3 a e 5a ordem (ou a 3a e 5a harmónicas) relativamente à onda fundamental. Consequentemente, oscilam com frequências de $180 \mathrm{~Hz}$ e $300 \mathrm{~Hz}$. Raciocínio análogo pode ser aplicado a outras ondas sinusoidais cujas frequências sejam outros múltiplos da frequência da onda fundamental [4].

Cargas que solicitam uma corrente que não toma uma forma de onda sinusoidal no tempo são denominadas de cargas não lineares. Assim, do ponto de vista da rede, a corrente elétrica a fornecer é não sinusoidal, ou seja, apresenta-se de acordo com o teorema de Fourier, uma soma de ondas sinusoidais com uma frequência de valor múltiplo da componente fundamental (componentes harmónicas).

Quando existem cargas não lineares ligadas à rede elétrica a corrente que circula nas linhas contém componentes harmónicas, e as quedas de tensão provocadas por estas nas impedâncias das linhas faz com que as tensões de alimentação fiquem também distorcidas, originando os harmónicos de tensão, sendo estes o resultado do produto da corrente harmónica vezes a impedância harmónica.

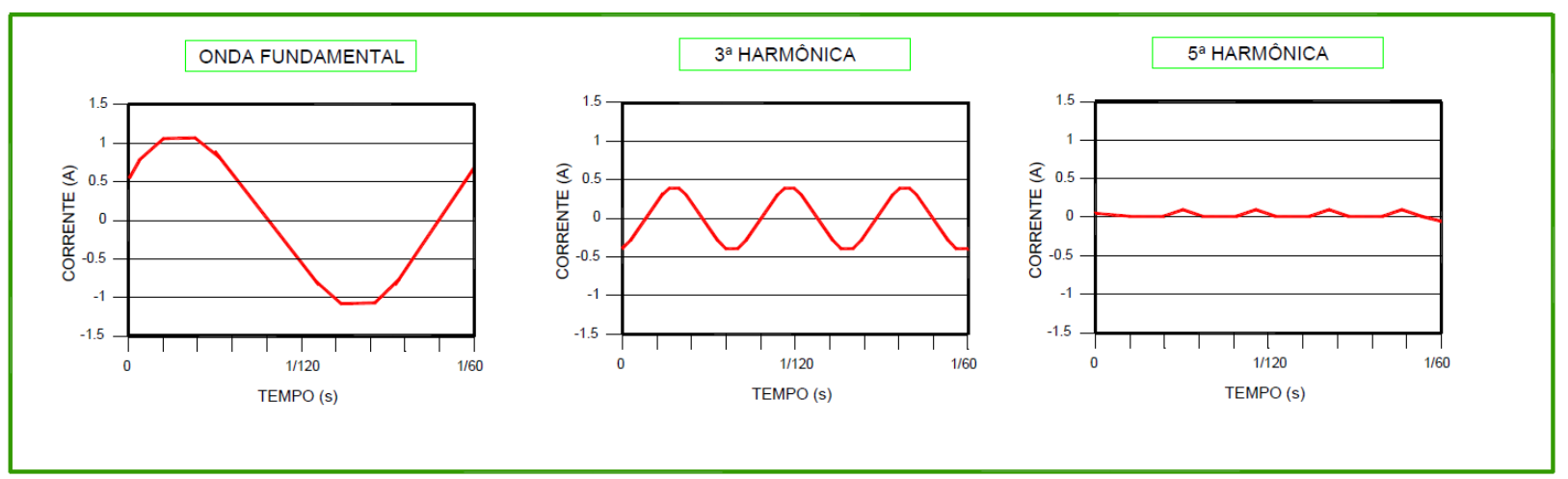

Figura 2. Exemplo de uma onda fundamental e da 3ạ e 5ạ harmónica [8] 
Se pretender-se reduzir as harmónicas de tensão, uma das maneiras possíveis é reduzir a impedância harmónica da rede a montante.

Nas instalações industriais é relevante falar ainda das "interharmónicas", que surgem quando há componentes de corrente que não são múltiplos inteiros da frequência da componente fundamental $(50 \mathrm{~Hz})$. Essas componentes de corrente podem ser produzidas por fornos a arco ou por ciclo-conversores, que são equipamentos que alimentados a $50 \mathrm{~Hz}$ permitem obter tensões e correntes de saída com uma frequência diferente.

\section{Indicadores de medição}

Uma medida muito usada da distorção harmónica é a Total Harmonic Distortion (THD). Esta é o quociente, expresso em percentagem, da raiz quadrada da soma dos quadrados das tensões eficazes de cada harmónica pelo valor eficaz da fundamental. $\mathrm{O}$ valor de THD é tanto maior quanto mais a forma de onda se afasta de uma sinusoide pura.

Considerando um sinal (pode ser a corrente ou a tensão), a taxa de distorção harmónica é definida pela fórmula abaixo:

$$
\text { THD } \%=100 * \frac{\sqrt{\sum_{n=2}^{N} y_{h}^{2}}}{y_{1}}
$$

Com a finalidade de conhecer a contribuição de cada harmónica, existe a distorção harmónica individual (DIT), segundo a expressão:

$$
D I T=\frac{y_{h}}{y_{1}}
$$

Fator de crista (FC) é a proporção entre o valor de pico de uma onda e o seu valor eficaz, considerando onda de corrente.

$$
F C=\frac{l_{\text {yico }}}{l_{r m s}}
$$

Essa relação é igual a $\sqrt{2}$ quando um sinal não possui distorção, ou seja, é perfeitamente sinusoidal.
Essa relação é igual a quando um sinal não possui distorção, ou seja, é perfeitamente sinusoidal.

Observando-se estas expressões, verifica-se que para a determinação dos valores das distorções harmónicas totais e/ou individuais presentes em uma determinada instalação elétrica, é preciso que sejam determinados os valores de cada componente harmónica individualmente, ou seja, torna-se necessário a realização de medições específicas.

\section{Causas}

As "cargas lineares" são as que obedecem à Lei de Ohm. Caracterizam-se por absorver da rede elétrica correntes proporcionais à tensão a elas aplicada, preservando-se as formas de onda sinusoidais, ainda que possa haver desfasamentos angulares entre elas. Note-se que para um elemento reativo, condensador ou bobina, haverá um desfasamento entre a tensão e a corrente, mas o comportamento ainda será linear. Qualquer carga que solicita uma corrente que não toma uma forma de onda sinusoidal no tempo é denominada de carga não linear e, de acordo com o teorema de Fourier, apresentará uma soma de ondas sinusoidais com uma frequência de valor múltiplo da componente fundamental (componentes harmónicas).

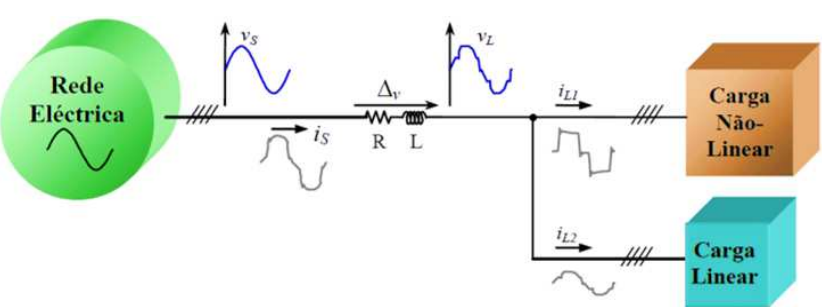

Figura 3. Harmónicas de Corrente/Tensão provocadas pela carga não linear [7]

Os tipos de cargas não lineares, fontes geradoras de componentes harmónicas presentes nos sistemas elétricos de potência, são bastante variadas, indo dos equipamentos dos sistemas produtores e fornecedores de energia, até aos consumidores, principalmente os industriais, que constituem uma parcela considerável da carga a ser alimentada, que inclui uma percentagem elevada da potência solicitada por cargas não lineares. 
No passado, as distorções harmónicas nos sistemas de potência eram primordialmente associadas à conceção das máquinas elétricas. Atualmente, com o uso generalizado de equipamentos eletrónicos, nomeadamente os baseados em eletrónica de potência (díodos, mosfets, tirístores, etc.) aumentou-se drasticamente as cargas não lineares que são alimentadas pelos sistemas elétricos de energia.

Sob o ponto de vista da rede de alimentação, os conversores estáticos de potência (retificadores e inversores) constituem a fonte mais expressiva de distorção harmónica. De facto, nas atuais instalações elétricas industriais, mais de metade da energia elétrica em cada momento (potência elétrica) passa por um dispositivo de eletrónica de potência antes que seja finalmente utilizada. Todos estes dispositivos de eletrónica de potência têm dois modos de funcionamento, condução que corresponde a um interruptor fechado ou bloqueio que corresponde a um interruptor aberto. A passagem de um estado para o outro é muito rápida, e em qualquer instante do sinal (através do controlo do semicondutor). Essas comutações rápidas de estado produzem uma corrente não sinusoidal, quando a tensão que alimenta os dispositivos é sinusoidal. Por sua vez, a circulação destas correntes não sinusoidais nas instalações e equipamentos elétricos conduz a quedas de tensão com evolução igualmente não sinusoidal, que quando sobrepostas adequadamente com a tensão da rede a tornam também não sinusoidal.

Os conversores estáticos de potência podem ser resumidos em 3 grandes grupos:

- Conversores de alta potência, usados em transmissão DC e na indústria siderúrgica;

- Conversores de média potência, usados para o controlo de motores em indústrias e tração ferroviária;

- Conversores de baixa potência (nomeadamente os retificadores), alimentandas cargas monofásicas, como aparelhos de televisão e carregadores de bateria.
Em adição a estes conversores, outras cargas não lineares como os compensadores estáticos reativos, os fornos elétricos a arco, cargas típicas industriais, constituem as restantes principais fontes de harmónicas para os sistemas e instalações elétricas. Em relação aos fornos a arco, equipamentos de solda (a arco voltaico no fundo) e à presença de ciclo-conversores (agora em desuso) verifica-se a presença de "inter-harmónicas" - são formas de ondas de tensões e correntes que apresentam componentes de frequência que não são múltiplos inteiros da frequência com a qual o sistema é suprido e designado a operar.

A figura 4 mostra a forma de onda de corrente absorvida por alguns tipos de cargas não lineares, tendo o espetro de distribuição em amplitude das harmónicas e a THD característica dessa forma de onda (corrente).

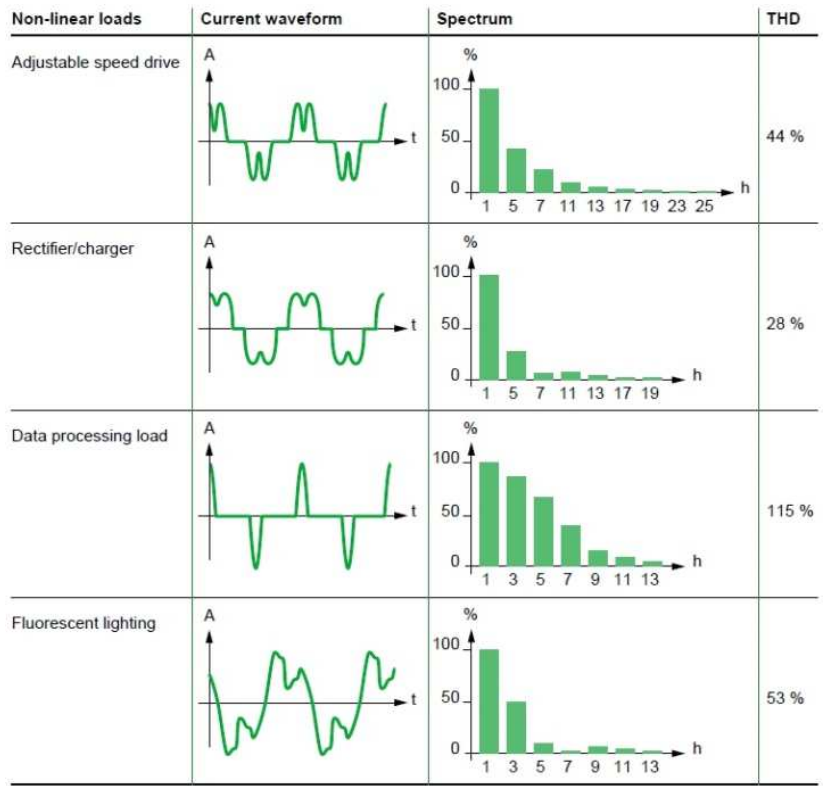

Figura 4. Cargas não lineares- Espetro e distorção harmónica $[5,8]$

\section{Consequências}

a) Fator de Potência

Com a presença de harmónicas numa instalação, o fator de potência baixa, acarretando vários problemas. 0 impacto mais percetível é o aumento das perdas na instalação e na rede elétrica, devido ao trânsito de potência na rede, e como consequência direta a diminuição da sua eficiência. $\mathrm{Na}$ definição habitual de fator de potência note-se que apenas é 
válida para formas de onda sinusoidais, ou seja, sem a presença de componentes harmónicas.

Definição habitual para ondas sinusoidais:

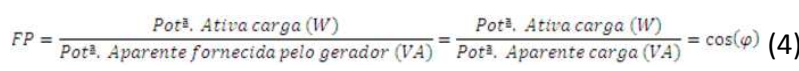

Definição geral para qualquer forma de onda:

$F P=\frac{\text { Potência Ativa carga }(W)}{\text { Potência Aparente fornecida pelo geradior }(V A)} \neq \cos (\varphi)$

Do desenvolvimento matemático, a partir da definição do fator de potência e dos valores eficazes da tensão e corrente decompostas em série de Fourier, pode-se chegar à seguinte expressão:

$$
F P=\frac{\cos \varphi}{\sqrt{1+T H D^{2}}}
$$

Deste modo verifica-se que um elevado conteúdo de componentes harmónicas prejudica o FP das instalações (maior THD). Um baixo fator de potência, como é visto por parte da rede quando existe um conteúdo de harmónicas elevado, resulta no aumento na corrente total circulante nas redes de distribuição de energia elétrica, podendo sobrecarregar as linhas de transmissão e transformadores, prejudicando a estabilidade e aproveitamento dos sistemas elétricos, acarretando problemas, tais como [6]:

- Perdas na rede e na instalação;

- Subutilização da capacidade instalada;

- Custos tarifários;

- $(\ldots)$.

\section{b) Máquinas Elétricas}

Os motores são sensíveis a distorções da tensão, ou seja, quando uma onda de tensão distorcida excita um motor, correntes de altas frequências estão a ser injetadas no estator. Por este facto, vão surgir componentes harmónicas de corrente que podem causar inúmeros problemas, como aquecimento, vibrações, binários pulsantes ou ruído.

Consequentemente, o aumento da temperatura dos motores reduzirá o tempo de vida médio dos mesmos. Como exemplo: nos alternadores, as harmónicas de corrente provocam perdas óhmicas suplementares nos enrolamentos principais e nos enrolamentos amortecedores. Por outro lado, a interação entre correntes harmónicas e o campo magnético fundamental, pode originar binários oscilatórios que provocam vibrações no veio dos alternadores e, consequentemente, o aumento da fadiga mecânica das máquinas.

Nos motores assíncronos ocorrem aumentos das perdas por efeito Joule, com o consequente sobreaquecimento dos enrolamentos estatóricos, além de que uma distribuição assimétrica da corrente induzida nas barras rotóricas provoca vibrações e o aparecimento de binários de torção no veio da máquina.

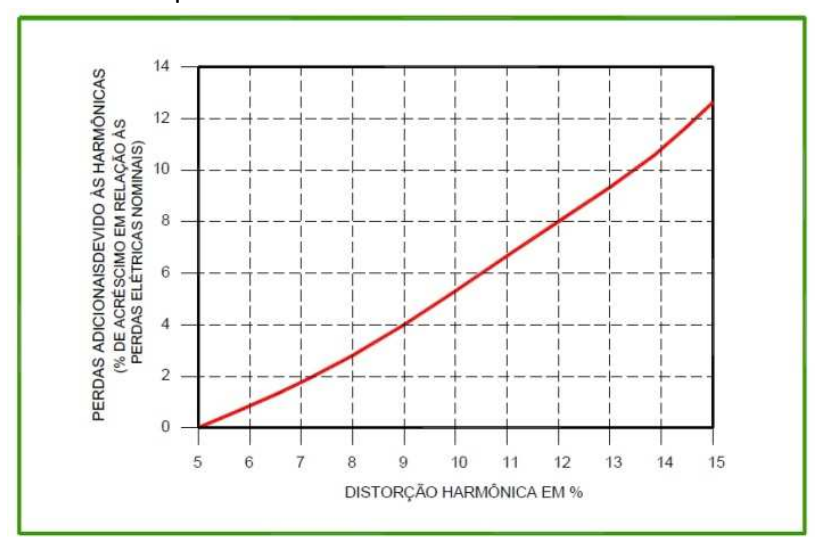

Figura 5. Perdas elétricas adicionais dos motores de indução [8]

\section{Situação no mercado}

Atualmente, existem empresas especializadas no setor que apresentam além de uma gama de produtos padronizada, um serviço personalizado que permite estudos e análise da qualidade de energia elétrica de uma qualquer instalação particular, permitindo conceber uma solução mais eficaz para o cliente, incluindo em muitos casos sistemas de compensação do fator de potência para ambientes harmonicamente poluídos, quando o sistema ideal de compensação da distorção harmónica for do ponto de vista económico pouco atrativo. 
Existem diversas técnicas para reduzir as componentes harmónicas de tensão e/ou corrente. De um modo global podem ser agrupadas nas estratégias assim caracterizadas:

- Uso de filtros passivos conectado sem paralelo e/ou em série com o sistema elétrico;

Os conversores estáticos de potência podem ser resumidos em 3 grandes grupos:

- Conversores de alta potência, usados em transmissão DC e na indústria siderúrgica;

- Aumento da quantidade de pulsos em unidades conversoras, com o uso de transformadores desfasadores;

- Técnicas de compensação de fluxo magnético

- Filtros ativos de potência ligados em paralelo e/ou em série com o sistema elétrico.

A escolha de um ou outro procedimento, ou mesmo a associação de soluções, deve levar em conta a análise dos seguintes aspetos:

- Conhecimento do sistema de alimentação do ponto de vista da concessionária: impedância de curtocircuito, nível de tensão e legislação quanto aos níveis de distorções harmónicas permitidos;

- Conhecimento do sistema consumidor: tipos de cargas instaladas, potência envolvida, problemas que ocorrem devido às harmónicas, perda de energia, diminuição do fator de potência real;

- Local da instalação do dispositivo para redução de harmónicas;

- Desempenho e capacidade nominal de tensão/corrente do dispositivo;
- Custo inicial de compra e custo da energia consumida no próprio dispositivo;

- Efeitos colaterais prejudiciais sobre o sistema de alimentação: o fator de potência em situações de carga nominal pode alterar-se em condições de carga baixa, modificação do nível e da distorção de tensão ou de corrente, alteração do nível de curto-circuito para a terra, mudança ou possibilidade de ressonância em outras frequências harmónicas. Em decorrência desses fenómenos, pode haver possíveis efeitos nocivos sobre outras cargas consumidoras adjacentes;

- Efeitos colaterais prejudiciais ao funcionamento das cargas elétricas envolvidas: aumento da distorção, abaixamento e elevação na tensão de alimentação da carga;

- Influências nocivas das variações do sistema sobre o dispositivo utilizado: alterações da impedância do sistema, correntes harmónicas de cargas consumidoras adjacentes podem entrar pela alimentação, o sistema pode desequilibrar-se em tensão, a distorção de tensão e o seu nível na barra de alimentação podem variar devido a fatores externos;

- Influência da carga sobre a técnica utilizada: a variação da potência solicitada pela carga e a presença de desequilíbrios podem alterar o funcionamento do dispositivo empregado para a redução de harmónicas.

A figura 6 demonstra que todas estas estratégias tendem a convergir para a atenuação máxima do problema, devendo ser combinadas sempre que possível, notando a importância do papel de todos os intervenientes, desde os fabricantes até aos utilizadores. 


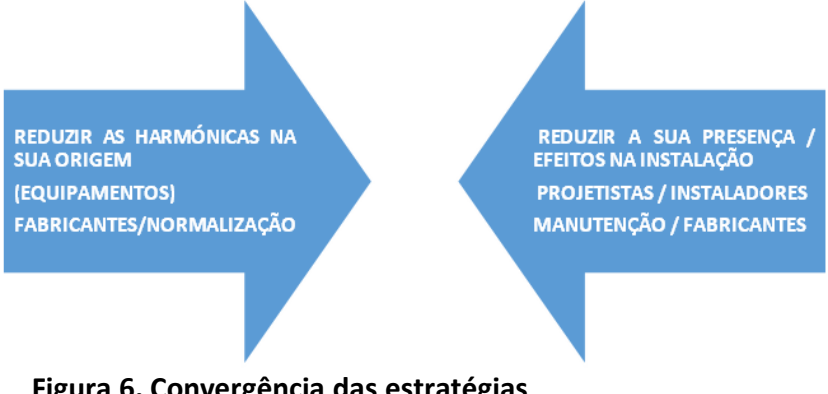

Figura 6. Convergência das estratégias

\section{Caso Prático}

Foi analisada uma instalação com base em dados obtidos através de equipamentos de medida instalados para o efeito. A amostragem de dados foi realizada num período de 2 dias. $\mathrm{Na}$ instalação em causa sabe-se que proliferam computadores pessoais e PLCs. Os dados que são apresentados, gráficos e tabelas, foram obtidos com recurso a uma aplicação informática que permite a visualização gráfica e tratamento de bases de dados. Os dados foram recolhidos registados e armazenados no equipamento de medida, um analisador de energia com aplicação informática.

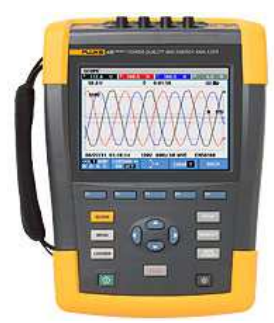

Figura 7. Analisador de energia [2]

Nas figuras abaixo apresentam-se os dados que serviram para a análise deste caso, recolhidos entre as $02 \mathrm{~h} 15$ e as 14h15 de 19-09-2013:

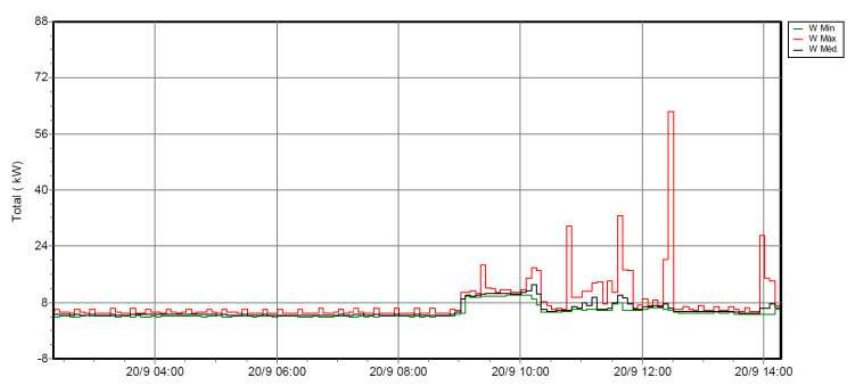

Figura 8. Potência Consumida
As distorções harmónicas são muito equiparadas nas 3 fases, razão pela qual só se inclui, na figura 9, os referentes à fase 1 .

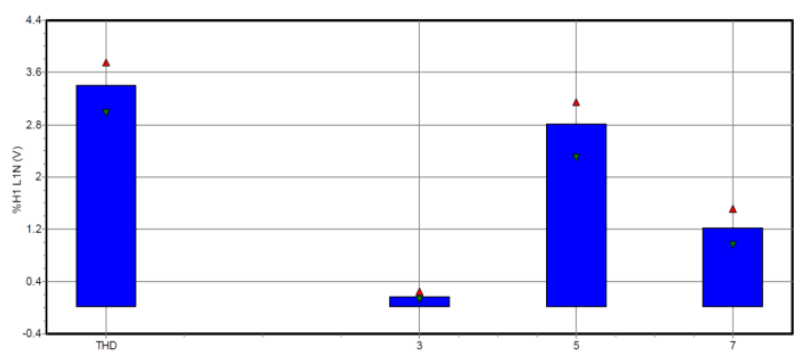

Figura 9. Distorção Harmónica de Tensão, L1N

A distorção harmónica também se manifesta no condutor de neutro, sendo a sua tensão mais de 3 vezes superior à tensão da frequência fundamental, ainda que a tensão do neutro obviamente não apresente valores muito elevados. As figuras 10 e 11 permitem identificar os valores apresentados.

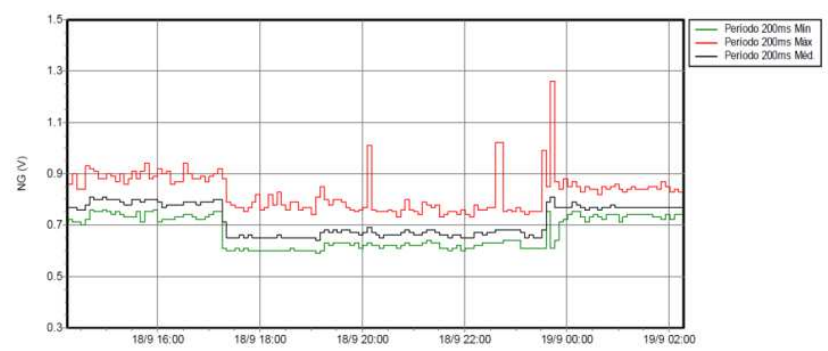

Figura 10. Tensão e Corrente

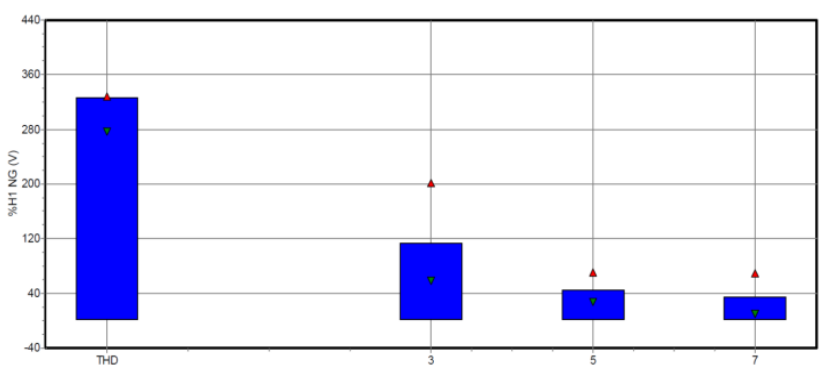

Figura 11. Distorção Harmónica de Tensão, NG

Como já foi referido, as componentes harmónicas são muito difíceis de combater, daí que apenas as mais importantes, ao nível da sua perturbação para os equipamentos, são alvo de atenção com vista à sua compensação. Nos dados das figuras 9 e 10 verifica-se a existência de perturbações referentes às harmónicas ímpares de 3, 5 e 7ạ ordem, sendo, por isso, importante a sua eliminação quando existam em valor percentual considerado grave, tendo em conta as normas em vigor e os custos que podem provocar. 
Segundo a NP EN 50160, o valor DTH total e individual nesta instalação e no período do registo não é preocupante, em virtude do seu valor ser inferior a 8 e $5 \%$, respetivamente. No entanto, poderá agravar-se, pelo que, para o bom funcionamento da instalação e respetivas cargas, é recomendável a supervisão por medição e análise continua.

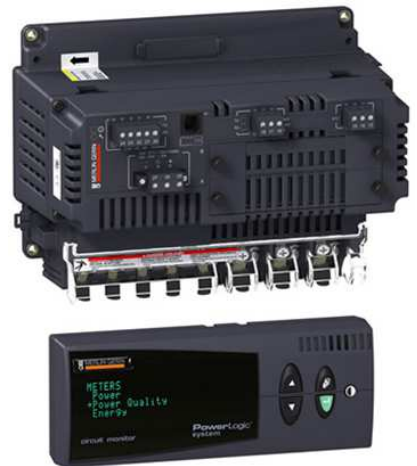

Figura 12. Centrais Monitorização DE QEE, CM3000/4000 [4]

Para a medição e análise de forma continuada, foram utilizados os equipamentos das figuras 7 e 12. Graças às suas funcionalidades, que incluem a deteção de fenómenos transitórios, pode-se resolver rapidamente os problemas associados à qualidade de energia, nomeadamente os relacionados com as componentes harmónicas.

Apesar dos dados registados não o evidenciarem, pode-se afirmar que existirão momentos na exploração desta instalação, ainda que pontuais e em determinadas horas de maior consumo de energia, em que as componentes harmónicas terão uma presença mais acentuada relativamente à que foi registada no período escolhido.

Atendendo que os custos de não produção são elevados, propôs-se como solução para o caso que é apresentado, além da supervisão por monitorização contínua, a compensação global a jusante do quadro geral com filtros ativos. Este equipamento compensará a distorção harmónica até ao limite do valor nominal, em qualquer frequência em que esta esteja presente, pelo que poderá começar-se por instalar um equipamento de entrada de gama 20 Amperes, garantindo na prática a eliminação na instalação de perturbações harmónicas durante a sua exploração.
A solução proposta vem de encontro às soluções mais comuns e eficazes disponíveis no mercado. Contudo, deve-se notar que é sempre aconselhado o dimensionamento do filtro adequado para cada caso específico, pois cada instalação tem as suas próprias características, assim como a rede que a alimenta.

Este será seguramente um investimento com um tempo de "Payback" reduzido, mesmo que apenas se contabilizem as perdas nos transformadores e condutores da instalação elétrica. Contudo, se adicionalmente forem também considerados os ganhos das avarias que serão evitadas nos equipamentos, será potencialmente fácil ao técnico responsável pela instalação convencer os órgãos de gestão na adoção destas soluções e na compra do respetivo equipamento.

\section{Conclusão}

Para a correta compreensão da problemática das perturbações harmónicas, à semelhança de grande parte de outras áreas da engenharia, o tratamento matemático é bastante relevante. As séries de Fourier são ajuda fundamental no conceito de definir e quantificar o que são as componentes fundamental e harmónicas.

O tema das "Perturbações Harmónicas" é complexo e extenso, conduzindo na maioria das vezes ao seu desconhecimento e há ausência de estudos detalhados, particularmente na fase de elaboração dos projetos das instalações elétricas. Os problemas, regra geral, surgem depois da entrada em serviço das instalações elétricas, sendo bastante mais complexa a análise e economicamente mais severas as consequências.

As soluções mais adotadas para minimizar os efeitos nefastos das distorções provocados pelas componentes harmónicas consistem geralmente na instalação de reactâncias, filtros passivos e filtros condicionadores ativos, na utilização de transformadores de isolamento, no reposicionamento e/ou alteração da potência de condensadores estáticos, na elevação da capacidade do sistema de alimentação, entre outras. 
A solução para uma determinada instalação é tão ou mais difícil quanto menor a quantidade de dados disponíveis para a análise da rede em questão. É muito importante a correta monitorização e análise tanto em termos de qualidade como de quantidade de dados obtidos.

De salientar que medidas, como por exemplo o correto dimensionamento de condutores (fases, neutro e terra), a separação de malhas de terra, a inclusão do "Fator K" (fator depreciativo determinado de acordo com a taxa de distorção harmónica espectável) no dimensionamento de transformadores para Postos de Transformação privados e públicos, assim como a implementação de baterias de condensadores para correção do fator de potência, devidamente dimensionadas para a taxa de distorção da instalação, são factores que não devem ser descorados no projeto de uma instalação elétrica, seja projeto de construção ou requalificação.

Neste documento, é evidenciado que as unidades industriais ou comerciais com forte utilização de equipamentos baseados em eletrónica de potência, tais como conversores de frequência, reactâncias eletrónicas, fontes comutadas, entre outros, são equipamentos passiveis de apresentar uma forte componente harmónica. Nestas unidades deve-se considerar sempre os efeitos das perturbações harmónicas, implementando medidas para o seu controlo e monitorização, de forma a limitar as eventuais perdas de produção e os respetivos custos associados a este problema.

\section{Bibliografia}

[1] Martinho, Edson, “ Distúrbios da Energia Elétrica”, Editora Érica, São Paulo, 2012, ISBN 978-85-365-0231-1.

[2] Sankaran, C., "PowerQuality - CRC Press", Estados Unidos da América, 2002, ISBN 0-8493-1040-7.

[3] Moreno, H., "Harmónicas nas Instalações Elétricas", Instituto Brasileiro do Cobre, São Paulo, 2001.

[4] Schneider Electric / Procobre, "Workshop Instalações Elétricas de Baixa Tensão: Qualidade de Energia Elétrica",

http://www.schneider-electric.com.br/documents/cadernostecnicos/harmon.pdf, consultado a 22 de Dezembro de 2013.

[5] Ferracci, Philippe - Cahiers Techniques - Schneider Electric's "Collection technique", Grenoble, 2000.

[6] SchneiderElectric, "Harmonics mitigation and solutions" http://www.schneider lectric.cn/medias/solutions/downloads/377ed18_harmonics _mitigation_solutions.pdf,consultado a 28 de Dezembro de 2013.

[7] Martins, Júlio, João, A., "Qualidade da Energia Elétrica" Revista o Eletricista, no 9, 3o trimestre de 2004, ano 3, pp. 66-71.

[8] Isoni, Marcos, “Dist orções Harmônicas - Uma Revisão de Conceitos Gerais", consultado a 11 de Dezembro de 2013.

[9] Schneider Electric, “Cahier technique no 199”, 2000, 